\title{
Phenomenological interpretation of internal erosion in granular soils from a discrete fluid-solid numerical model
}

\author{
L. Sibille \\ Université Grenoble Alpes, 3SR Laboratory, F-38000 Grenoble, France \\ D. Marot \& P. Poullain \\ Nantes Université, GeM Institut, IUT de St-Nazaire, 44600 Saint-Nazaire, France \\ F. Lominé \\ INSA Rennes, Laboratoire de Génie Civil et Génie Mécanique 35000 Rennes, France.
}

\begin{abstract}
Internal erosion in granular soils may involve different steps: the detachment of solid particles from the granular skeleton under the action of water seepage; the transport of the detached particles carried with the water flow in the pore space; and eventually, for some erosion processes, such as suffusion, the possible reattachment of some transported particles to the solid skeleton of the soil, acting as a filter. The first part of this paper is devoted to the description and interpretation of the first step about the particle detachment. The analysis is mainly based on direct numerical simulations performed with a fully coupled discrete element-lattice Boltzmann method. Dynamics of the solid granular phase is represented thanks to the discrete element method in which each solid particle is explicitly described, whereas dynamics of the interstitial water flow is solved with the lattice Boltzmann method. Interactions between the solid phase and the fluid phase are handled at the particle scale avoiding the introduction in the model of some phenomenological constituents to deal with fluid-solid interactions. Numerical modellings of hole erosion can be interpreted similarly to laboratory hole erosion tests where the erosion rate is linearly related to the hydraulic shear stress. Further investigations from the numerical results suggest that the erosion rate for hole erosion in granular soil, can also be interpreted as a function of the water flow power according to a power law. The latter interpretation is applied to experimental data from suffusion tests on a cohesionless soil and glass bead mixtures. Here again, if change of erosion rate due to filtration is discarded, erosion rate is correctly described by the water seepage power according to a power law. Finally, a simple phenomenological model is suggested to describe the whole suffusion process, based on the previous results, to describe the particle detachment, and completed to take also into account the transport and filtration phases. Predictions of this model are compared with experimental results from suffusion tests on glass bead mixtures.
\end{abstract}

\section{INTRODUCTION}

Four main types of internal erosion can be identified in water-retaining structures made of soil (Fell \& Fry 2007, Bonelli 2012): the concentrated leak erosion (erosion of a crack or a hole), the backward erosion (progressing from the surface of the downstream part of the structure), the suffusion (taking place in the soil bulk), and contact erosion occurring at an interface between fine and coarse soil layers respectively. In all these processes of internal erosion, soil particles are firstly detached by the action of the water flow and secondly transported in the existing hole (or crack), or the pore network of the soil. A third step of self filtration may occur in the case of suffusion (or even for contact erosion (Béguin 2011)) when transported particles are redeposited within the interstitial space of the soil (possibly leading to a partial clogging of the latter (Reddi et al. 2000, Sail et al. 2011)).

Hydraulic shear stress is classically considered in the cases of contact erosion and concentrated leak erosion (as hole erosion) as the hydraulic loading parameter fixing the erosion regime (Béguin et al. 2013, Wan and Fell 2004, Bonelli and Brivois 2008, Haghighi et al. 2013). Concerning the erosion by suffusion the development of suffusion has been described in the literature from different hydraulic parameters such as the global (Skempton \& Brogan 1994) or local (Moffat \& Fannin 2006) hydraulic gradient, the hydraulic shear stress (Reddi et al. 2000), or 
the pore fluid velocity (Perzlmaier 2007).

Nevertheless, a look at the work in the field of sediment transport in river beds and overland flows shows that several authors use the stream power (representing an estimation of the power dissipated by viscous shear in the fluid near the fluid/soil interface) to characterize solid particle detachment and/or transport (Bagnold 1956, Bagnold 1980, Low 1989, Govers 1992, Ferro 1998). More recently, attempts have also been made in the field of soil internal erosion to characterize the hydraulic loading driving the erosion development by the flow power (the power consumed by the water to seep through the soil) for suffusion (Marot et al. 2012, Rogoz 1985) and hole erosion (Regazzoni and Marot 2013, Marot et al. 2011). A possible advantage of such an approach could be constituted by the rather simple estimation of the flow power through a soil sample or a soil structure (a dam or a dike for instance) from the water head drop and the seepage flow rate (Rochim 2015).

In this paper, we take advantage of recent developments in coupling numerical discrete element model (DEM) with computational fluid dynamics to perform direct numerical simulations with a hole erosion model in order to investigate the possibility to characterize internal soil erosion from the flow power. In the numerical model, the DEM, used to describe the motion of each particle of a granular soil, is coupled with the lattice Boltzmann method to solve the dynamics of the interstitial fluid flow (Lominé et al. 2013). With such a numerical model, assumptions about fluidsolid interactions are considerably reduced and very few mechanical parameters are introduced. That is why we are speaking about direct simulations (or numerical experiments). The numerical model is presented in a first section of the paper. Then interpretations of the particle detachment step is discussed in a second section, from the numerical data, in terms of both hydraulic shear stress and flow power. Then we attempt in a last section to apply the expression identified from the numerical experiments to a case of erosion by suffusion characterized in laboratory on glass bead mixtures. As in suffusion transported particles may be filtrated, contrary to the hole erosion, the description is completed to take into account this latter step characterizing the suffusion.

\section{DETACHMENT OF SOLID PARTICLES / INITIATION OF EROSION}

\subsection{A micro hydro-mechanical model of hole erosion}

Numerical experiments of hole erosion have been performed with a two-dimensional coupled DEM-LBM numerical model (cf. Figure 1). The solid granular phase is described with the discrete element method in which each solid particle is represented with a circular disk. Contact forces between two particles are computed according to simple contact laws and can be decomposed into a normal contact force, $F_{n}$, normal to the contact plane, and a shear contact force, $F_{s}$, tangent to the contact plane. The relation between $F_{n}$ and the normal component of the relative displacement of particles at contact point is purely elastic. The relation between $F_{s}$ and the relative shear (or tangential) displacement is also elastic up to a sliding limit represented by a Coulomb criterion defined by a contact friction angle $\phi_{c}$. Beyond this limit the contact is sliding in the tangential direction and energy is dissipated by dry friction. Contact strength can be increased by considering additional normal and shear cohesion terms, $C_{n}$ and $C_{s}$, as represented in Figure 2. Fluid dynamics is solved between solid particles with the lattice Boltzmann method (LBM) with a relatively fine discretization of the fluid domain (the ratio of the mean solid particle diameter to the fluid lattice space is about 17). The coupling between the LBM and the DEM is based on the balance of momentum between the fluid and the solid phase computed around each boundary of solid particles (Lominé et al. 2013). Not any assumption is introduced in the definition of coupling (except the no slip condition at the boundary between the two phases). Consequently, the mechanical parameters introduced in the coupled method are very limited: the fluid viscosity, and the parameters defining the contact interaction law between solid particles (stiffnesses, cohesions and friction angle). Hence, for instance, hydraulic conductivity of drag forces constitute output, and not input, of the numerical method.

The bi-dimensional model is displayed in Figure 1 (Lominé et al. 2013). It consists of a tube filled, in its central part, of solid grains, circular and cohesive, characterized by a mean diameter $d_{\text {mean }}$. The grains along the tube axis have been manually removed in order to create an initial hole with an aperture $d_{h} \approx 4 d_{\text {mean }}$. The contact generated in the initial configuration between two grains, or between a grain and a wall of the tube, are cohesive. Normal and shear cohesions, $C_{n}$ and $C_{s}$ (defined in Figure 2 are chosen such that $C_{n}=C_{s}=C$. The cohesion is brittle, hence if cohesion breaks, contacts become purely frictional (see Figure 2). Finally, any new contact created during the simulation is itself purely frictional.

The tube walls are impermeable with respect to the fluid and constitute a rigid boundary for the solid particles. A pressure drop $\Delta P$ is imposed between the inlet and the outlet of the tube to generate the fluid flow through the hole, with a Reynold number from 0.50 to 40.0 . The effect of gravity is discarded. These simulations reproducing about $200 \mathrm{~s}$ of physical time, and including 800 solid grains and more that 300,000 fluid nodes, were performed for 10 different values of pressure drop, and 7 values of contact cohesion.

Under the action of the fluid flow, solid grains are detached from the boundary of the hole and carried with the fluid until the outlet section of the tube (Figure 3). The grains crossing the latter section are con- 


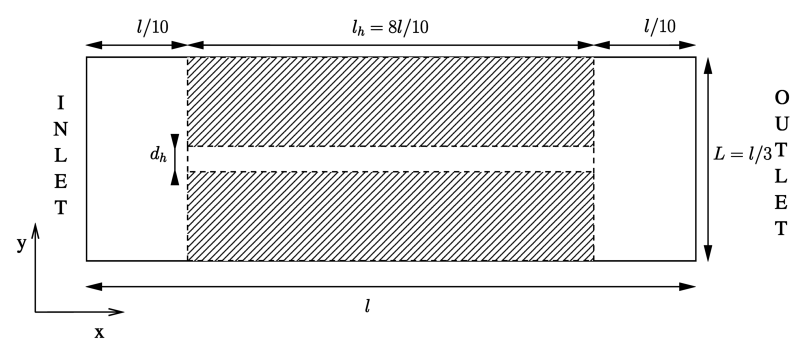

Figure 1: Sketch of the numerical model of hole erosion; hatched regions correspond to the locations of the cohesive granular assembly such that an initial horizontal hole is formed

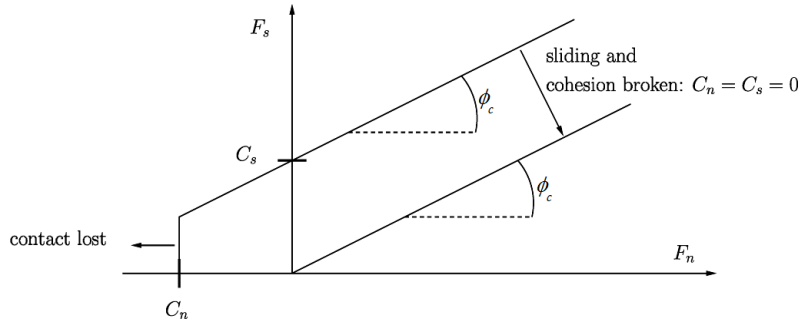

Figure 2: Failure criterion of the cohesive brittle contacts

sidered as eroded. $M_{e}$ denotes the cumulated mass of eroded grains. Typical time series of the eroded mass ratio $M_{e} / M_{0}$ (where $M_{0}$ is the initial mass of the granular assembly) are presented in Figure 4.

\subsection{Particle detachment and hydraulic shear stress}

The classical description of the hole erosion (or concentrated leak erosion) consists in representing the hydraulic loading by the hydraulic shear stress $\tau_{s}$ developing on the solid/fluid interface of the hole. Then the erosion rate per unit surface $\dot{\epsilon}$ depends on the excess shear stress with respect to the critical stress below which particle detachment does not occur (Wan \& Fell 2004):

$\dot{\epsilon}=k_{d}\left(\tau_{s}-\tau_{c}\right) \quad$ if $\quad \tau_{s}>\tau_{c}$

where $k_{d}$ is an erosion coefficient fixing the kinetics of particle detachment for shear stresses exceeding the threshold. It is worth noting that such a description of particle detachment driven by the hydraulic shear stress has been also considered in the case of the erosion by suffusion by Bonelli \& Marot (2011), who represented the pore of soils as small tubes. Then, they assumed solid particles are detached from the peripheral surface of the tubes as in a hole erosion problem.

Results obtained from the numerical model and presented in Figure 5 are in agreement with this interpretation. A parametric study with respect to the contact cohesion $C$ showed the critical stress for particle detachment increases with $C$, apparently linearly; whereas the erosion coefficient $k_{d}$ seems independent of $C$ (Sibille et al. 2015a). However, these conclusions should be confirmed by further studies involving a wider range of variation of $C$ values.

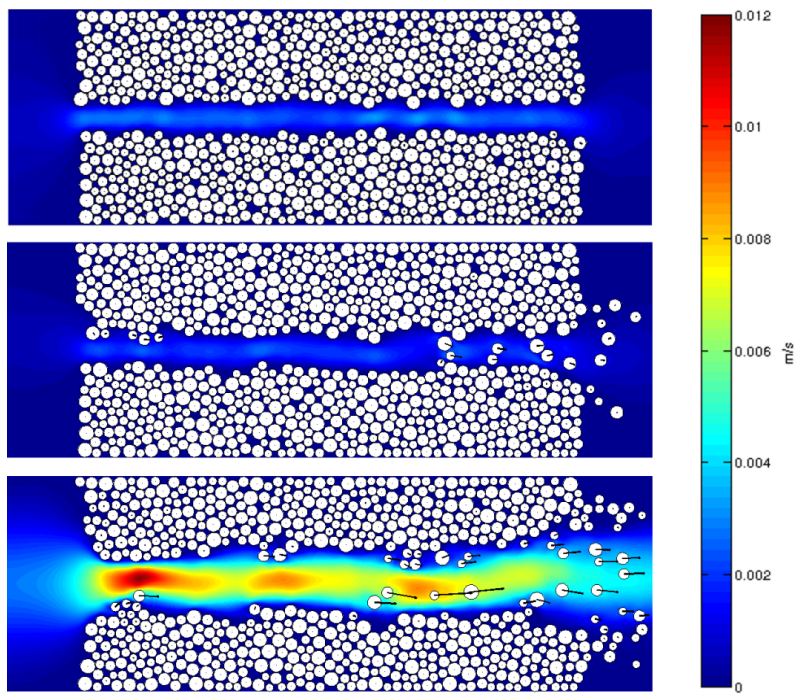

Figure 3: Snapshots of a simulation of erosion (at $t=0.7,13.3$ and $55.0 \mathrm{~s}$ from top to bottom), the colour scale is related to the fluid velocity whereas the translation velocity of solid particles is represented with arrows

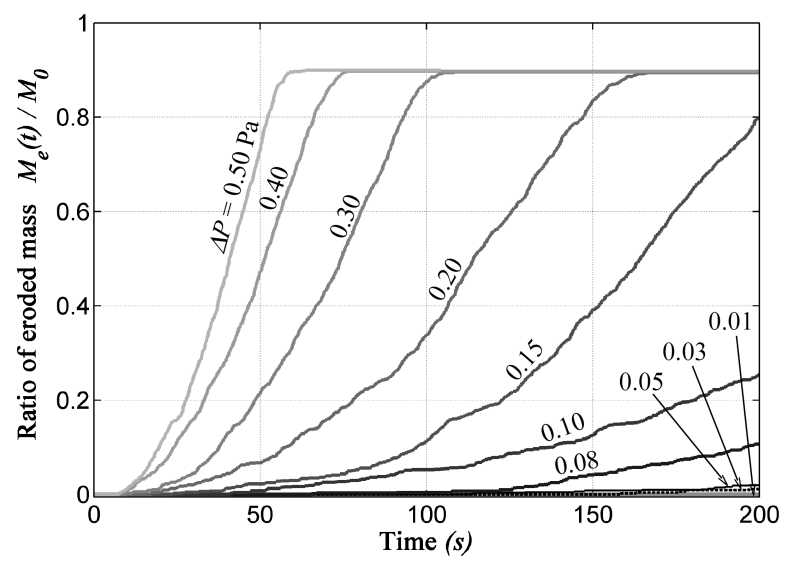

Figure 4: Eroded mass for a given cohesion $(C / d=0.506 \mathrm{~N} / \mathrm{m})$ simulated for pressure drops ranging from $\Delta P=0.01$ to 0.50 $\mathrm{Pa}$

\subsection{Particle detachment and fluid flow power}

The use of flow power (or stream power) to characterize the transport of sediments in river beds has been suggested since the 50s by Bagnold (1956, 1980), but also more recently for overland flow sediment transport (Low 1989, Govers 1992, Ferro 1998). In both cases (river and overland flow), the stream power is defined as the product between the hydraulic shear stress on the bed soil and the mean flow velocity, giving an estimation of the power dissipated by viscous shear in the fluid near the soil bed interface. Bagnold (1980) and Govers (1992) showed that the sediment transport rate can be expressed as a power function of the stream power. Recently, Marot et al. (2012) and Regazzoni \& Marot (2013) suggested that internal erosion in soils could be driven by the power dissipated by the fluid seepage throughout the porous solid phase. Therefore, we investigate in this section the possibility to interpret the internal erosion of soils from energetic terms representative of the water flow through the soil.

Nevertheless, it is generally accepted that internal erosion in soils comprises three steps: detachment of 


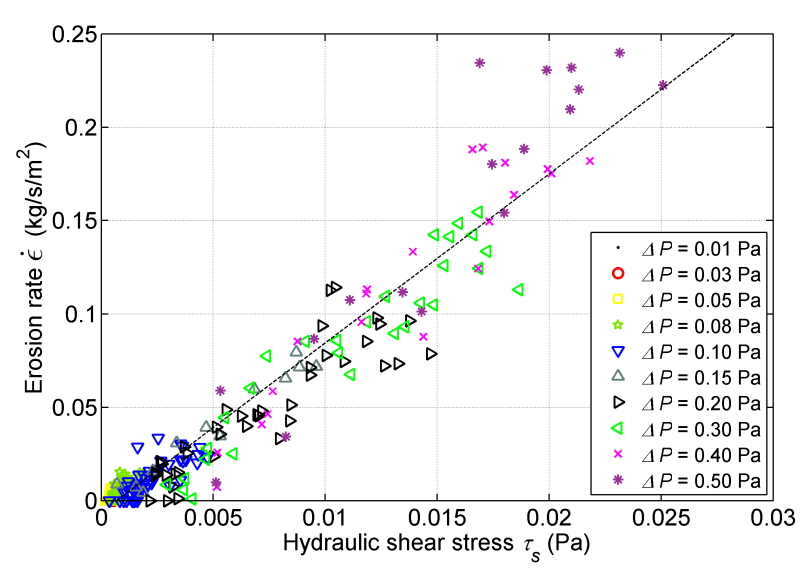

(a)

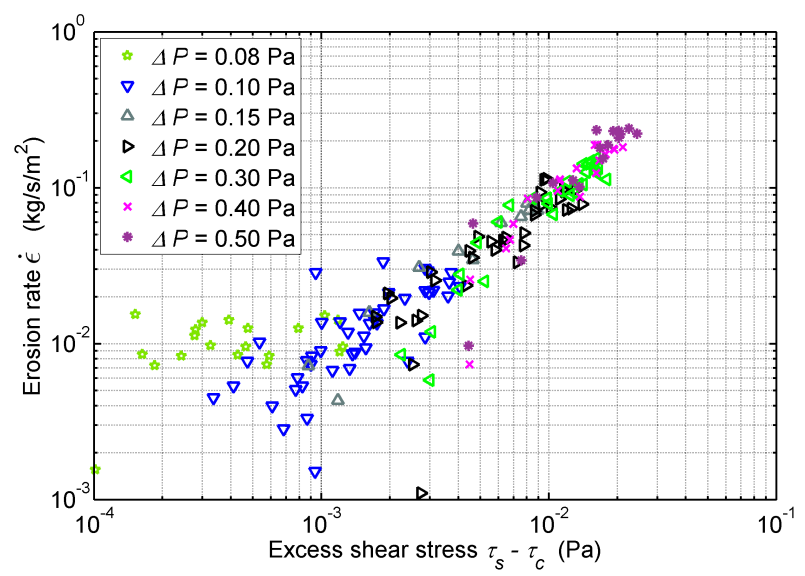

(b)

Figure 5: Simulated erosion rate for a cohesion $C / d=0.506 \mathrm{~N} / \mathrm{m}$ in terms of the hydraulic shear stress estimated on hole boundaries, (a) linear scale and (b) logarithmic scale (in the latter the excess shear stress $\tau_{s}-\tau_{c}$ is used instead of the shear stress and points with shear stress values lower than the threshold $\tau_{c}$ have been removed)

solid particles from the initial granular skeleton, their transport within the interstitial space and eventually a possible deposition (or filtration) of the transported particles in soil pores. In the framework of sediment transport in rivers and overland flow, there is no filtration step and the interpretation in terms of stream power introduced above aims to characterize only the detachment and transport of particles. Consequently, the analysis carried out here after in the framework of internal erosion, is voluntarily limited in a first time to the step of detachment for the sake of separation of problems. The discrete numerical model has indeed been designed to avoid deposition or filtration, because even if some particles may temporarily settle, detachment and transport are far more predominant with an irreversible increase of the hole width.

\subsubsection{Fluid power of a seepage through a granular medium}

Let us consider an incompressible viscous fluid. We define at a position $\vec{x}$ of its volume $V$, the fluid density $\rho$, its static pressure $p$ and velocity $\vec{v}$, and the tensor of viscous stresses $\overline{\bar{\sigma}}_{v}$. The energy conservation equation of a volume $V$ of fluid seeping through solid grains in a tube (Figure 6) delimited by inlet and outlet surfaces, $S_{i}$ and $S_{o}$ respectively, writes (without taking into account the gravity) (Sibille et al. 2015a):

$$
\begin{aligned}
-\int_{S_{i}+S_{o}} p \vec{v} \cdot \vec{n} d S & =\int_{S_{G}}\left[p \vec{v} \cdot \vec{n}_{G}-\left(\overline{\bar{\sigma}}^{v} \cdot \vec{n}_{G}\right) \cdot \vec{v}\right] d S+ \\
& +\int_{V} \sigma_{i j}^{v} \frac{\partial v_{i}}{\partial x_{j}} d V
\end{aligned}
$$

with: $\vec{n}$ the outer unit normal vector to inlet and outlet surfaces, and $\vec{n}_{G}$ the outer unit normal vector to the boundary surface of solid grains.

The left-hand side of equation (2) represents the power supplied to the fluid to flow within the granular assembly. This term is called the flow power $P_{F}$. The integral over surface $S_{G}$, that will be denoted $I_{G}$, represents the power transferred from the fluid to the solid particles, and the remaining volume integral is the power dissipated by viscous stresses in the fluid bulk $P_{V}$. Then: $P_{F}=I_{G}+P_{V}$.

One could be interested in describing the action of the interstitial fluid flow on the solid phase of the soil from the term $I_{G}$. From a study on laboratory tests of suffusion and hole erosion it seems difficult to estimate the proportion of the flow power transferred to the solid phase to deform it and detach some particles, because this transfer represents a quasi negligible fraction in suffusion case and is highly variable in hole erosion cases (Sibille et al. 2015a).

Consequently we paid attention to the flow power $P_{F}$ which can be easily estimated, in an engineering context, from a flux and a pressure drop. In addition, the flow power is mainly dissipated by viscosity (term $P_{V}$ ) in the neighborhood of the solid interfaces (constituted by the solid grains included in the intact granular skeleton and the detached grains currently transported by the fluid), at least for the seepage configurations we studied (see Figure 7). Hence the flow power $P_{F}$ can be seen as an indicator of the fluid-solid interactions.

\subsubsection{Description of particle detachment from the flow power}

In the framework of the lattice Boltzmann method, we defined the fluid boundary nodes (FB nodes) as the

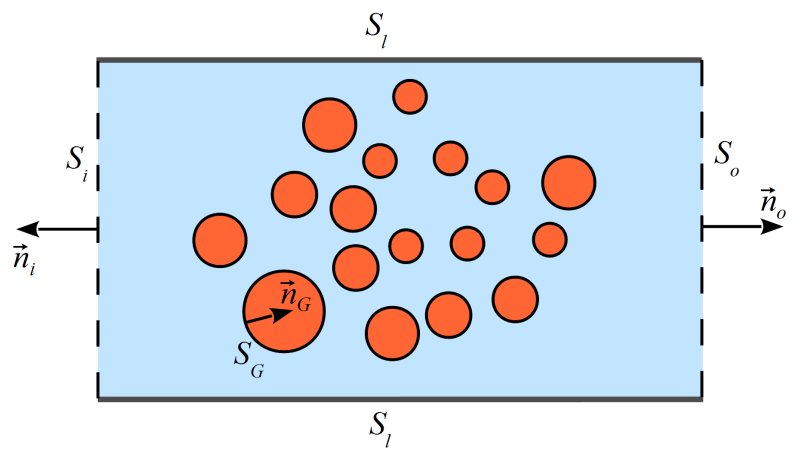

Figure 6: Water seepage through a granular assembly enclosed in a tube: the boundaries of the fluid domain comprise outer boundaries (inlet and outlet sections $S_{i}$ and $S_{o}$, and lateral tube surface $S_{l}$ ) and inner boundaries $S_{G}$ formed by the solid grains 

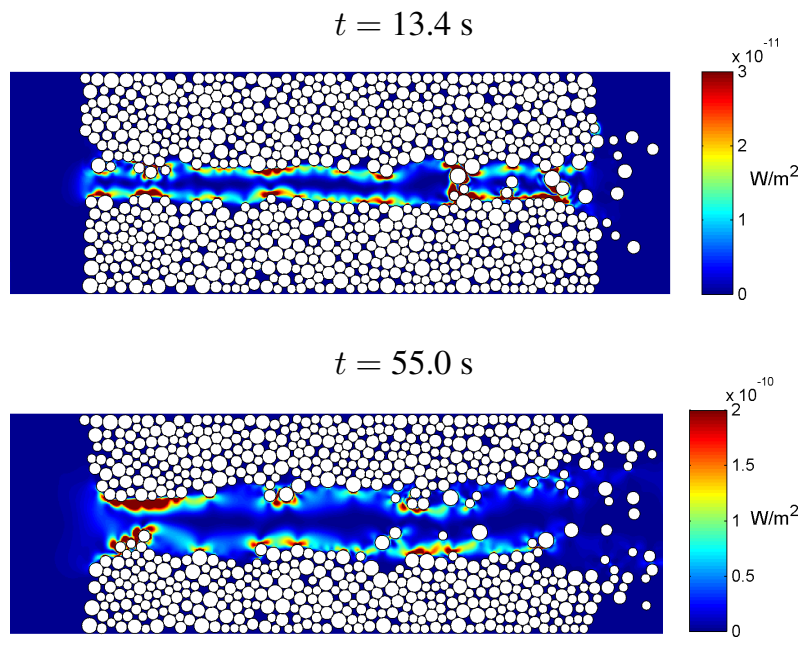

Figure 7: Field of density of power dissipated by viscosity (cohesion $C / d=1.27 \mathrm{~N} / \mathrm{m}$ and pressure drop $\Delta P=0.30 \mathrm{~Pa}$ )

computational nodes of the fluid domain constituting its boundary on the fluid/solid interface (Lominé et al. 2013). Hence, the power dissipated by viscosity at FB nodes, $P_{V}^{F B}$, represents the power dissipation occurring the most closely with the solid particles. The plot of the erosion rate in terms of $P_{V}^{F B}$ in Figure 8a shows the erosion rate varies as a power function of the excess power dissipated by viscosity at the direct vicinity of solid particles:

$\dot{m} \propto\left(P_{V}^{F B}-P_{V}^{F B *}\right)^{\beta_{V}}$

where $P_{V}^{F B *}$ is a threshold power below which particle detachment does not occur.

$P_{V}^{F B}$ cannot be directly quantified from a practical point of view. Then an identical interpretation can be carried out by considering the total flow power $P_{F}$ as displayed in Figure 8b. Although the scaling of the erosion rate with $P_{F}$ is slightly less satisfying than with $P_{V}^{F B}$, the erosion rate can also be expressed satisfyingly by:

$\dot{m} \propto\left(P_{F}-P_{F}^{*}\right)^{\beta_{F}}$

where $P_{F}^{*}$ constitutes a threshold value for particle detachment. In this last case the hydraulic loading represented by the flow power $P_{F}$, computed as the product between the fluid flux and the pressure drop, is quite easy to determine.

It is worth noting that imposed pressure drops, and thus fluid velocities, have been kept voluntarily small in these numerical simulations leading to a flow regime characterized by a Reynolds number between 0.50 and 40.0. Such a flow regime may be quite far from what is expected in hole erosion tests, usually characterized by a Reynolds number from 2,000 up to 20,000 (Bonelli 2012). This point constitutes a restriction of the numerical model. Nevertheless such an interpretation, of particle detachment for a hole erosion, based on flow power, has been satisfyingly applied on experimental data from a laboratory hole erosion test (Sibille et al. 2015a).

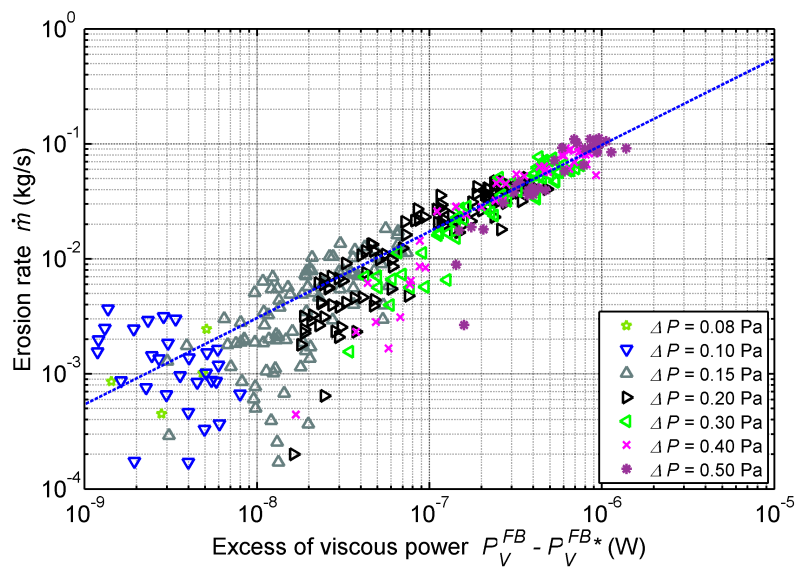

(a)

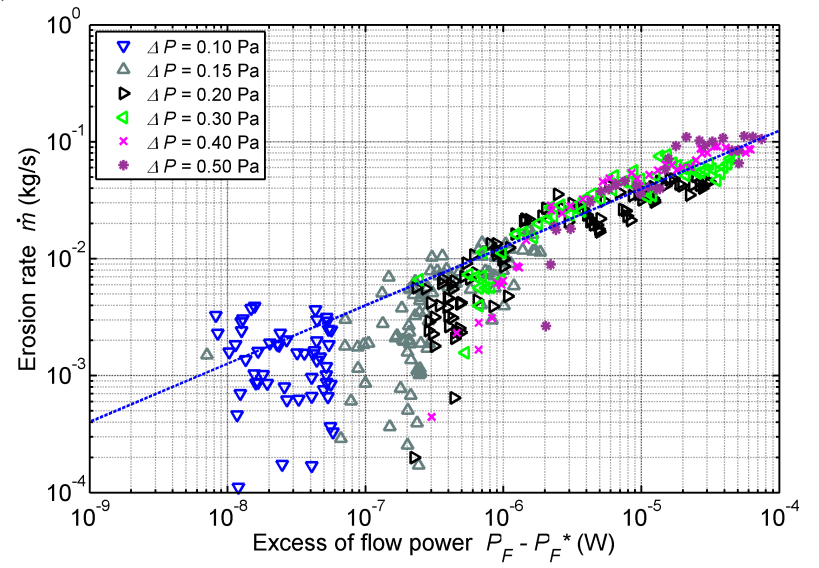

(b)

Figure 8: (a) Erosion rate as a function of the power dissipated by viscosity at fluid boundary nodes $P_{V}^{F B}$ (for a cohesion $C / d=$ $1.27 \mathrm{~N} / \mathrm{m}$ ), the dashed line represents an approximation with a power law; (b) similar interpretation but in terms of the flow power $P_{F}$.

\section{DESCRIPTION OF INTERNAL EROSION INCLUDING A FILTRATION STEP}

We assume here, that the description of particle detachment based on the flow power, presented in the previous section holds in the case of erosion by suffusion of granular materials (Bonelli \& Marot 2011). In other words it is assumed the mechanisms of detachment of particles involved in the suffusion are similar to the one represented with the numerical model of hole erosion. Nevertheless, suffusion may be accompanied by an additional step of filtration, possibly dominating the step of particle detachment, and limiting the erosion of particles after a given time.

Suffusion tests were carried out on a glass bead mixture composed of small beads (diameter ranging from 0.1 to $0.2 \mathrm{~mm}$ ) and bigger beads (diameter ranging from 1.2 to $3.4 \mathrm{~mm}$ ). This is therefore a gapgraded material with fines (small beads) constituting $40 \%$ of the total mass. Tests were performed with a oedo-permeameter sketched in Figure 9 and described by Sail et al. (2011). The sample made of beads is built inside the rigid cell of the oedo-permeameter, above a wire mesh with $1.25 \mathrm{~mm}$ pore opening size avoiding the migration of the biggest particles. The sample is then compressed under a vertical stress of $25 \mathrm{kPa}$ and slowly saturated with an upward flow. 
Table 1: Properties of suffusion tests on glass bead assemblies.

\begin{tabular}{llll}
\hline Test & $\begin{array}{l}\text { Initial sample length } \\
(\mathrm{mm})\end{array}$ & $\begin{array}{l}\text { Successive increments of global hydraulic gradient } \\
(-)\end{array}$ & $\begin{array}{l}\text { Duration of hydraulic loading increments } \\
\text { (hours) }\end{array}$ \\
\hline N1 & 250 & $1-2-3-3.2$ & $1-1-1-1$ \\
N2 & 450 & $1-2-3-4-4.8$ & $1-1-1-1$ \\
N3 & 450 & $1-2-3-3.7-4.7$ & $1-1-1-1$ \\
N4 & 600 & $1-2-3-4.9-5.5$ & $1-1-1-1$ \\
N5 & 250 & $0.1-0.2-0.4-0.8-1-2.2-3$ & $1-1-1-1-1$ \\
N6 & 250 & $0.1-0.2-0.4-0.8-1$ & $1-1-5-4-1$ \\
\hline
\end{tabular}

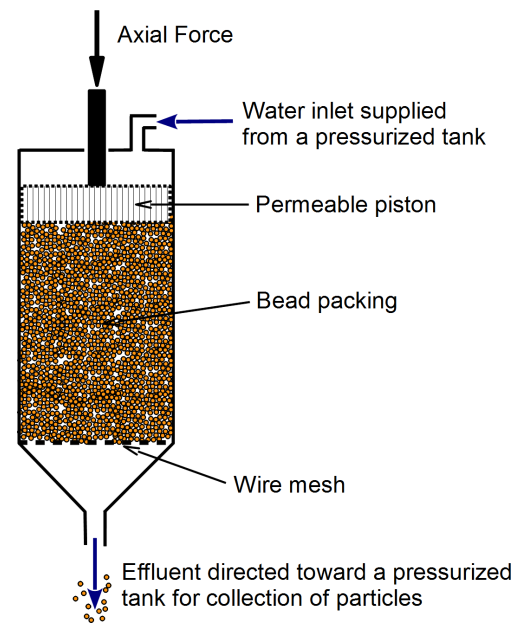

Figure 9: Sketch of the oedo-permeameter used to perform suffusion tests on glass bead assemblies

Once the saturation phase is finished the bead samples are subjected to a downward water flow under a controlled hydraulic gradient $i$ increasing by steps. An erosion test consists in successive steps of about 60 min (except for test N6) with a constant hydraulic gradient $i$. Tests are presented in the Table 1 and discussed in detail in (Sibille et al. 2015b). They differ from the size of the increments of hydraulic gradient between each step, and the length of the tested samples.

Beads carried with the effluent at the outlet of the cell are collected using a sampling device (Sail et al. 2011). Time series of the cumulative mass of collected beads are shown in Figure 10. Steps of the hydraulic gradient are visible, since at the beginning of each step, mass increases rapidly and tends to stabilize at the end of the step. The decreasing of the erosion rate during a hydraulic step is related to the limitation of detachable fine beads (this limitation being itself dependent on the hydraulic gradient (Bonelli \& Marot 2011), and the possible development of filtration (re-deposition of transported particles within the sample itself). Consequently, we assume that only the erosion rate at the initiation of each step of hydraulic gradient is characteristic of particle detachment. Erosion rate per unit volume, $\dot{\bar{m}}$, is plotted according to the flow power per unit volume, $\bar{P}_{F}$, for the six tests (N1 to N6) in Figure 11. The highest erosion rates displayed in the latter plot correspond to the initiation of increments of hydraulic gradient and are assumed to be representative of the detachment of solid particles, independently of the quantity of potentially erodible
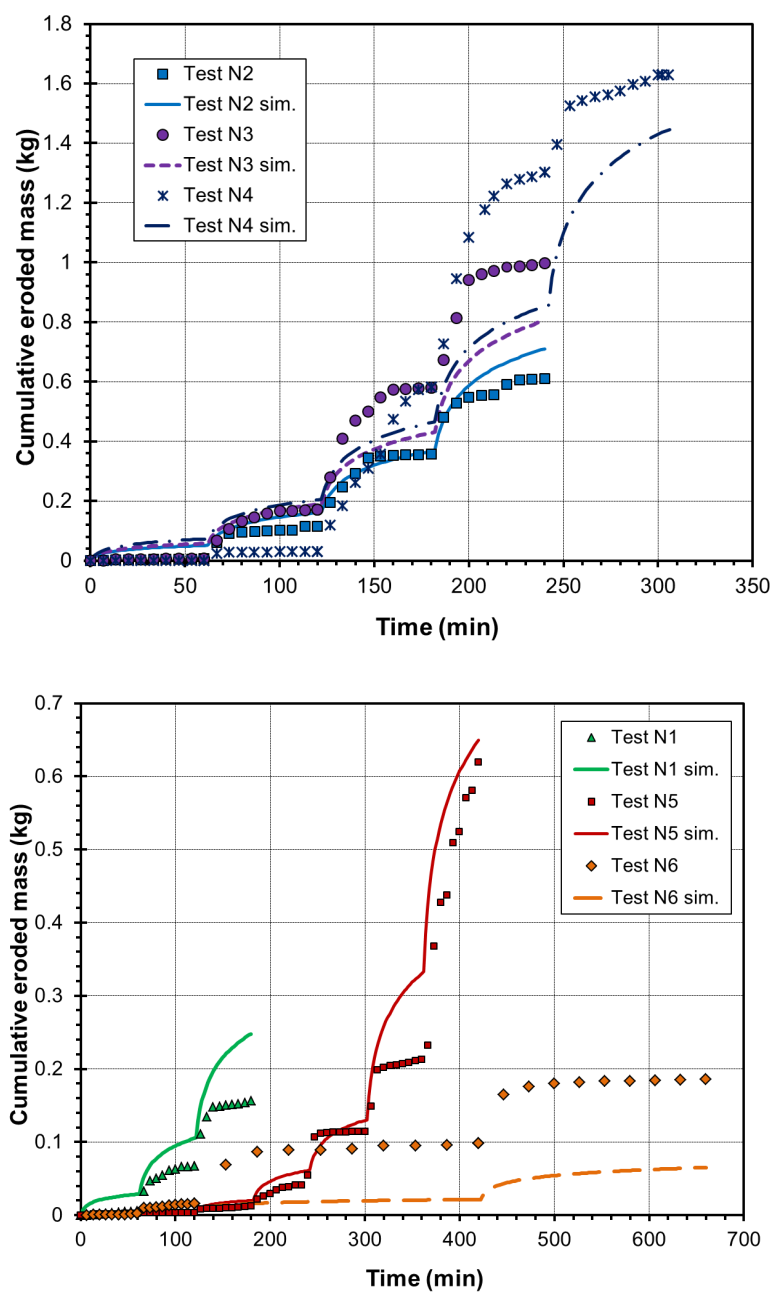

Figure 10: Cumulative eroded mass produced from suffusion tests on glass bead mixtures, symbols represent experimental data whereas continuous lines represent a model prediction

beads and of a possible filtration. This is represented by the upper limit envelop of data plotted with the dashed line in Figure 11 and approximated, as suggested in equation (4), with the power law :

$\dot{\bar{m}}^{\text {upper limit }}=\alpha_{\text {ref }}\left(\bar{P}_{F}\right)^{\beta_{F}}$

with $\alpha_{\text {ref }}=0.003$ and $\beta_{F}=0.8$, which can be seen as intrinsic parameters to the tested material and representing its erodability. The threshold flow power $P_{F}^{*}$ is very low for this kind of material and as been neglected here for the sake of simplicity.

The decrease in erosion rate during each step of hydraulic gradient emphasizes the necessity to take into account the history of hydraulic loading (the amplitude but also the duration of each increment). Then 


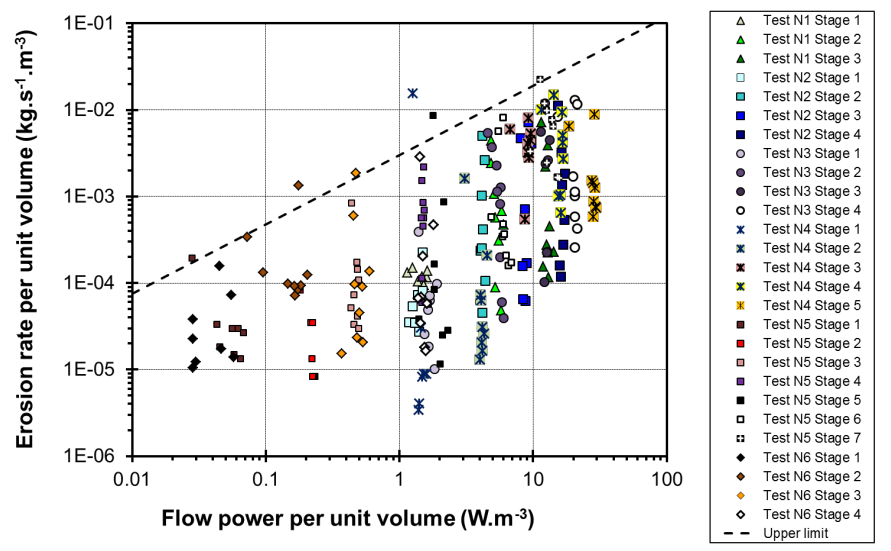

Figure 11: Identification of the maximum erosion rate per unit volume as a function of the flow power per unit volume

we define a history parameter, the cumulated flow energy per unit volume $\Delta \bar{E}$ equal to the time integration of the instantaneous flow power, $\bar{P}_{F}$, from the initiation of the considered increment of hydraulic gradient. Taking into account this history parameter we propose the following expression of the mass erosion rate (Sibille et al. 2015b):

$\dot{\bar{m}}=\alpha_{\text {ref }} \frac{1}{\frac{\Delta \bar{E}}{\bar{P}_{F} t^{*}}+1}\left(\bar{P}_{F}\right)^{\beta_{F}}$

where $t^{*}$ is a characteristic time relative to the material (taken here equal to $130 \mathrm{~s}$ by calibration from test N2). For the tests discussed here, the hydraulic conductivity of the material was only slightly affected by the erosion. Consequently, by assuming the hydraulic conductivity constant and equal to $k_{0}$, the one initially measured on the glass bead assembly, the erosion rate per unit volume, $\dot{\bar{m}}$, can be computed at any time. The cumulative eroded mass is directly deduced from the latter, integrated over the time, and compared with the experimental data in Figure 10. The description proposed is able to capture the main features of the erosion process. However, the prediction of eroded mass is not totally in agreement with the experimental data. Although tests N2 and N3 have been performed with the same parameters (see Table 1) and thus stand for the repeatability of tests, the cumulative mass of particles collected is about $25 \%$ larger for test N2 than N3. Obviously, the model is not able to describe such a difference since the input parameters are identical (or at least almost identical with an initial hydraulic conductivity $k_{0}=1.24 \cdot 10^{-4} \mathrm{~m} / \mathrm{s}$ for $\mathrm{N} 2$, whereas $k_{0}=1.50 \cdot 10^{-4} \mathrm{~m} / \mathrm{s}$ for test N3). Consequently, due to the discrepancies between the experimental data, it is difficult to conclude here about the ability of prediction of the model. Nevertheless it is worth noting this model stays rather simple, involving four parameters $\left(\alpha_{\text {ref }}, \beta_{F}, t^{*}, k_{0}\right)$ identifiable from a single suffusion test performed by increasing the hydraulic gradient by steps.

\section{CONCLUSION}

An alternative description of hole erosion was suggested on the basis of direct numerical simulations performed with a coupled DEM-LBM model. This interpretation states the detachment rate of solid particles can be represented from the seepage power according to a power law. This is not in opposition with a more common interpretation based on the hydraulic shear stress, since the seepage power mainly depends on the work of the hydraulic shear stress at the vicinity of fluid-solid interfaces. However, a rough determination of the seepage power can be easily assessed from a drop of water head and an estimation of the seepage flow rate. This can present an advantage for the application of this interpretation in engineering problems and in the field.

This description has been then extended to erosion by suffusion from the interpretation of suffusion tests performed on glass bead mixtures. At the initiation of suffusion, detachment and transport of solid particles can also be described from the seepage power according to a power law. Additional terms are needed for larger time periods to take into account the finite quantity of potentially erodible particles (with respect to the hydraulic loading applied) and the filtration step. This results in a simple and rough phenomenological model involving four parameters. This model is able to capture the main features observed experimentally.

\section{REFERENCES}

Bagnold, R. (1956). The flow of cohesionless grains in fluids. Philosophical Transactions of the Royal Society of London. Series A, Mathematical and Physical Sciences 249(964), 235-297.

Bagnold, R. (1980). An empirical correlation of bedload transport rates in flumes and natural rivers. Proc. R. Soc. Lond. A 372(1751), 453-473.

Béguin, R. (2011). Etude multi-échelle de l'érosion de contact au sein des ouvrages hydrauliques en terre. $\mathrm{Ph}$. D. thesis, Université de Grenoble.

Béguin, R., P. Philippe, \& Y. Faure (2013). Pore-scale flow measurements at the interface between a sandy layer and a model porous medium: Application to statistical modeling of contact erosion. J. Hydraulic Engineering 139(1), 1-11.

Bonelli, S. (Ed.) (2012). Erosion in Geomechanics Applied to Dams and Levees. ISTE-Wiley.

Bonelli, S. \& O. Brivois (2008). The scaling law in the hole erosion test with a constant pressure drop. Int. J. Numer. Anal. Meth. Geomech. 32(13), 1573-1595.

Bonelli, S. \& D. Marot (2011). Micromechanical modeling of internal erosion. European Journal of Environmental and Civil Engineering 15, 1207-1224.

Fell, R. \& J. Fry (Eds.) (2007). Internal erosion of dams and their foundations. Taylor \& Francis Publisher.

Ferro, V. (1998). Evaluating overland flow sediment transport capacity. Hydrological Processes 12, 1895-1910.

Govers, G. (1992). Evaluation of transporting capacity formulae for overland flow. In A. Parsons and A. Abrahams (Eds.), Overland flow, pp. 243-273. UCL Press, London.

Haghighi, I., C. Chevalier, M. Duc, S. Guédon, \& P. Reiffsteck (2013). Improvement of hole erosion test and results on ref- 
erence soils. Journal of Geotechnical and Geoenvironmental Engineering 139(2), 330-339.

Lominé, F., L. Scholtès, L. Sibille, \& P. Poullain (2013). Modelling of fluid-solid interaction in granular media with coupled lb/de methods: application to piping erosion. Int. J. Numer. Anal. Meth. Geomech. 37(6), 577-596. doi: 10.1002/nag.1109.

Low, H. (1989). Effect of sediment density on bed-load transport. Journal of Hydraulic Engineering 115, 124-138.

Marot, D., V. Le, J. Garnier, L. Thorel, \& P. Audrain (2012). Study of scale effect in an internal erosion mechanism: centrifuge model and energy analysis. European Journal of Environmental and Civil Engineering 16(1), 1-19.

Marot, D., P. Regazzoni, \& T. Wahl (2011). Energy-based method for providing soil surface erodibility rankings. $J$. Geotech. Geoenviron. Eng. 137(12), 1290-1293.

Moffat, R. \& J. Fannin (2006). A large permeameter for study of internal stability in cohesionless soils. Geotechnical Testing Journal 29(4), 273-279.

Perzlmaier, S. (2007). Hydraulic criteria for internal erosion in cohesionless soil. In R. Fell and J.-J. Fry (Eds.), Internal erosion of dams and their foundations, pp. 179-190. Taylor \& Francis.

Reddi, L., I. Lee, \& M. Bonala (2000). Comparison of internal and surface erosion using flow pump test on a sand-kaolinite mixture. Geotechnical Testing Journal 23(1), 116-122.

Regazzoni, P. \& D. Marot (2013). A comparative analysis of interface erosion tests. Natural hazards 67(2), 937-950. doi:10.1007/s11069-013-0620-3.

Rochim, A. (2015). Characterization of suffusion susceptibility of granular soils. Ph. D. thesis, Université de Nantes, Nantes - St-Nazaire.

Rogoz, M. (1985). Seepage power as a factor determining the piping and silting up the loose water-bearing soils. In R. Fernández-Rubio (Ed.), Proceedings, 2nd International Mine Water Association Congress, Volume 1, Granada, pp. 221-229. School of Mines, Department of Hydrogeology.

Sail, Y., D. Marot, L. Sibille, \& A. Alexis (2011). Suffusion tests on cohesionless granular matter. European Journal of Environmental and Civil Engineering 15(5), 799-817.

Sibille, L., F. Lominé, P. Poullain, Y. Sail, \& D. Marot (2015a). Internal erosion in granular media: direct numerical simulations and energy interpretation. Hydrological Processes 29(9), 2149-2163.

Sibille, L., D. Marot, \& Y. Sail (2015b). A description of internal erosion by suffusion and induced settlements on cohesionless granular matter. Acta Geotechnica 10(6), 735-748.

Skempton, A. \& J. Brogan (1994). Experiments on piping in sandy gravels. Géotechnique 44(3), 440-460.

Wan, C. \& R. Fell (2004). Investigation of internal erosion and piping of soils in embankment dams by the slot erosion test and the hole erosion test. UNICIV Report 412, University of New South Wales. 